\title{
To determine the prevalence of dyslipidemia and lipoprotein abnormalities among siblings of young acute myocardial infarction patients.
}

\author{
Awadhesh Kumar Sharma ${ }^{*}$, Ranjit Kumar Nath', Neeraj Pandit ${ }^{2}$ \\ ${ }^{1}$ Department of Cardiology, LPS Institute of Cardiology, Kanpur, UP, India \\ ${ }^{2}$ Department of Cardiology, Atal Bihari Vajpayee Institute of Medical Sciences (ABVIMS) and Dr. Ram Manohar \\ Lohia Hospital, New Delhi, India
}

\begin{abstract}
Introduction: Despite all available theoretical assumptions, there is no study defining the prevalence of lipid and lipoprotein abnormalities among siblings of young acute myocardial infarction (MI) patients. This is the first such study.

Methods: This was a case-control study. A total 110 siblings of young acute MI patients (Group A) and 50 healthy young controls (Group B) were studied for a duration of 2 years. Clinical profiles included age, sex, smoking, hypertension, diabetes mellitus, abdominal obesity and dyslipidemia for both cases and controls.The primary objective was to study the prevalence of dyslipidemia and lipoprotein abnormalities. The secondary objective was to study the prevalence of conventional risk factors among siblings of young ( $<45$ years) acute MI patients.

Results: On analyzing lipid profiles, it was found that total cholesterol, triglycerides, low-density lipoprotein, ApoB100, ApoB100: ApoA-1 ratio, and lipoprotein (a) were significantly raised in Group $A$ in comparison to Group B. On studying conventional risk factors, it was observed that history of smoking, hypertension, diabetes mellitus, and increased waist circumference were more prevalent in Group A in comparison to Group B.

Conclusion: Conventional atherosclerotic risk factors, lipid and lipoprotein abnormalities were significantly more prevalent in siblings of young acute MI patients in comparison to healthy controls and may be an answer to the possible cause of familial clustering in young MI emphasizing the importance of familial screening. Therefore, intensive efforts should be made to identify and alter modifiable risk factors in these cases.
\end{abstract}

Keywords: Acute coronary syndrome, Apolipoproteins, Dyslipidemia, Lipoprotein (a), Myocardial infarction.

Accepted on June 26, 2020

\section{Introduction}

Nowadays coronary heart disease (CHD) is the main cause of death all over the world due to sedentary lifestyle [1,2]. The age group above 45 years is the most widely affected by acute myocardial infarction (MI). However, nowadays due to stressful life attributable to psychological, social and financial constraints, persons below 45 years presenting with acute MI are no exception [3]. The main causes for increasing incidence of these events in the younger age group are physical inactivity, smoking, unhealthy diet, obesity, stressful life with overlapping appointments, and family history [4]. The cut-off age of 45 has been used in most studies to define young patients with MI and the same age criteria was used in this study [5].

Family and twin studies are consistent in showing the strong influence of genetic factors on premature coronary artery disease (CAD) [6,7]. Several prospective studies indicate that a family history of premature CHD is an independent risk factor even when other risk factors are taken into account [8-10]. The relative risk for CHD in first-degree relatives has been reported to be as high as $2-12$ times that of the general population [11-13].
Familial clustering may be due to genetic resemblance between members of the family, common family environment, or a probable contribution of both factors. Members of a family will tend to share similar environmental factors such as occupational classification, diet, smoking, exercise habits, and psychosocial stress, which have been shown to be related to the incidence of ischemic heart disease. This will perhaps be true of siblings rather more than of parent and child [14].

A large US study of persons developing CHD before the age of 60 years showed that low-density lipoprotein (LDL) cholesterol concentration of $>130 \mathrm{mg} / \mathrm{dL}$ was more than twice as common in asymptomatic siblings under the age of 60 years as in the population at large (38\% vs. 16\%) [15]. Analogous but much less pronounced differences were observed in the European Atherosclerosis Research Study (EARS) [16] which investigated young adults with a paternal history of MI before the age of 55 years. In this study the best lipoprotein discriminants were plasma ApoB100 and triglyceride concentrations, which were higher in those with a positive family history of premature CHD compared to age and sex matched controls. 
Citation: Sharma AK, Nath RK, Pandit N. To determine the prevalence of dyslipidemia and lipoprotein abnormalities among siblings of young acute myocardial infarction patients. Curr Trend Cardiol 2020;4(1):24-28.

\section{Methods}

\section{Study design and patient population}

This case-control study was conducted at the Department of Cardiology of two premier medical institutes. A total of 123 siblings of young (age $<45$ years) Indian patients with acute MI and 62 healthy young controls were screened for a duration of 2 years. Out of the 123 siblings, 110 (Group A) were selected based on the inclusion and exclusion criteria and out of 62 healthy controls, 50 (Group B) were selected based on the inclusion and exclusion criteria. All patients provided written informed consent.

\section{Study inclusion and exclusion criteria}

Siblings of young (age $<45$ years) Indian patients with acute MI were included in the study. Patients with (a) acute inflammatory illness (within the last month); (b) valvular heart disease; (c) liver disease; (d) renal failure; (e) acute infectious diseases; (f) adherence to anti-hyperlipidemia drugs; and (g) unwillingness to comply with study protocol were excluded from the study.

\section{Study definitions}

Baseline clinical characteristics of siblings of young (age $<45$ years) acute MI patients were evaluated. Clinical profiles included age, sex, smoking history, hypertension, diabetes mellitus, abdominal obesity and dyslipidemia for both cases and controls. Smokers were defined as patients who either smoked a cigarette daily or with a history of smoking within 2 years before study inclusion. Non-smokers were defined as patients who had not smoked a cigarette in his/her life or had quit smoking at least 2 two years before inclusion in the study [17]. Hypertension was diagnosed in those patients adhering to anti-hypertensive medication or with either systolic blood pressure of $\geq 140 \mathrm{mmHg}$ or diastolic blood pressure of $\geq 90$ $\mathrm{mmHg}$ recorded at least twice during standard examination [18]. Diabetes mellitus was diagnosed in those patients with (a) fasting blood glucose of $\geq 126 \mathrm{mg} / \mathrm{dL}$ at least twice at admission; (b) 2-hour plasma glucose $\geq 200 \mathrm{mg} / \mathrm{dL}$ during an OGTT (75 g); (c) random plasma glucose $\geq 200 \mathrm{mg} / \mathrm{dL}$ with symptoms of hyperglycemia; (d) treatment with hypoglycemic agents or insulin; or (e) hemoglobin A1c (HbA1c) level of $6.5 \%$ (48 $\mathrm{mmol} / \mathrm{mol})$ or higher [19]. Central obesity was defined as per waist circumference measurements. Men and women with waist circumference measurements of $>102$ and $>88 \mathrm{~cm}$, respectively, were considered to have high waist circumference [20]. Dyslipidemia was diagnosed according to Adult Treatment Panel (ATP) III criteria. It was diagnosed if plasma lipid analysis showed one or more of the following: hypercholesterolemia (total cholesterol $: \geq 200 \mathrm{mg} / \mathrm{dL}$ ) and/or hypertriglyceridemia (triglycerides: $\geq 150 \mathrm{mg} / \mathrm{dL}$ ), and low levels of high-density lipoprotein (HDL-C: $\leq 40 \mathrm{mg} / \mathrm{dL}$ in males and $\leq 50 \mathrm{mg} / \mathrm{dL}$ in females) [21]. LDL values were said to be increased if they were: (a) $\geq 100 \mathrm{mg} / \mathrm{dL}$ in presence of CAD or CAD risk equivalent; $(b) \geq 130 \mathrm{mg} / \mathrm{dL}$ in presence of $\geq 2$ risk factors; or $(\mathrm{c}) \geq 160 \mathrm{mg} / \mathrm{dL}$ in presence of $0-1$ risk factors. Lipoprotein abnormalities were considered if ApoB100 was found to be $>130 \mathrm{mg} / \mathrm{dL}$, Apo A-1 was found to be $<120$ $\mathrm{mg} / \mathrm{dL}$ in males and $<140 \mathrm{mg} / \mathrm{dL}$ in females. The ApoB100: ApoA-1 ratio $>0.6$ and $\mathrm{Lp}(\mathrm{a})>14 \mathrm{mg} / \mathrm{dL}$ were considered to be significantly associated with increased risk of CHD. Criteria for acute, evolving, or recent MI was typical rise and/or fall of biochemical markers of myocardial necrosis with electrocardiographic changes indicative of ischemia (STsegment elevation or depression) [22].

\section{Procedures of measurement}

Venous blood was collected from the antecubital vein of patients after overnight fasting. Individuals were seated for at least 5 minutes prior to phlebotomy to avoid hemoconcentration. $4 \mathrm{ml}$ of blood was collected in a plain vial for routine biochemical investigations and extended lipid profile. Serum extracted after centrifugation was stored in aliquots at $-20^{\circ} \mathrm{C}$ and not thawed till batch analysis for serum Apo A-1, ApoB and Lp(a) was completed. Analysis was done within 6 months as per stability suggested in the literature provided with the kit. Lipid profile, Apo A-1, ApoB, and Lp(a) testing and measurement was done by auto analyzer. Apo A-1 and ApoB measurement was done by Roche Tina-quaint Apolipoprotein A-1 reagent and Roche Tina-Quant Apolipoprotein B reagent using an immunoturbidometric assay on Roche/Hitachi 917 analyzer.

\section{Study objectives}

The primary objective was to determine the prevalence of dyslipidemia and lipoprotein abnormalities among siblings of young (age $<45$ years) acute MI patients. The secondary objective was to determine the prevalence of conventional risk factors such as smoking, hypertension, diabetes mellitus, and abdominal obesity among siblings of young (age $<45$ years) acute MI patients.

\section{Statistical analysis}

Statistical quantitative data were analyzed using the unpaired ttest or Mann-Whitney U test for comparisons of data between the different patient groups. For qualitative variables chisquare test or Fischer's exact test was applied. A p-value of $<0.05$ was considered statistically significant. For statistical analysis Graph pad Instat 3 software system was used.

\section{Results}

\section{Conventional risk profile}

There was no significant difference in age and gender between Group A and Group B. Mean age of study population in Group $A$ and Group B was $38.20 \pm 5.7$ years and $35.76 \pm 7.9$ years, respectively $(\mathrm{p}=0.06)$. The total number of male patients in Group A was $77(70 \%)$ and in Group B was $36(71 \%)(p=0.87)$ while the number of female patients in group A was $33(30 \%)$ and in Group B was $14(29 \%)(p=0.86)$. Conventional risk factors such as smoking, hypertension, diabetes mellitus, and increased waist circumference were statistically more prevalent 
in group A in comparison to group B as demonstrated in Table 1 .

Table 1. Conventional risk factor distribution in both groups.

\begin{tabular}{|l|l|l|l|}
\hline \multicolumn{1}{|c|}{ Risk factors } & \multicolumn{1}{|c|}{$\begin{array}{c}\text { Group A } \\
(\mathbf{n}=110)\end{array}$} & $\begin{array}{c}\text { Group B } \\
(\mathbf{n}=50)\end{array}$ & \multicolumn{1}{|c|}{ p value } \\
\hline Smoking & $80(72 \%)$ & $26(52 \%)$ & 0.021 \\
\hline Hypertension & $69(62 \%)$ & $15(30 \%)$ & 0.0003 \\
\hline Diabetes mellitus & $42(38 \%)$ & $6(12 \%)$ & 0.001 \\
\hline Increased waist circumference & $67(60 \%)$ & $16(32 \%)$ & 0.001 \\
\hline
\end{tabular}

All data are expressed as number (percentage)

\section{Lipid profile abnormalities}

On analyzing lipid profile among patients in both Groups A and $\mathrm{B}$, it was found that mean total cholesterol, triglycerides, LDL, and HDL of cases of Group A were significantly different from the controls of Group B. Mean total cholesterol among cases (Group A) was $210.09 \pm 38.11 \mathrm{mg} / \mathrm{dL}$, which was significantly greater than controls (Group B) which was 186.62 $\pm 31.67 \mathrm{mg} / \mathrm{dL} \quad(\mathrm{p}=0.0001)$. Similarly, mean triglycerides among cases (Group A) was $162.82 \pm 25.08 \mathrm{mg} / \mathrm{dL}$ which was greater than controls (Group B) which was $145.36 \pm 27.83$ $\mathrm{mg} / \mathrm{dL}(\mathrm{p}=0.0002)$. On the same lines, mean LDL cholesterol among cases (Group A) was $140.31 \pm 39.27 \mathrm{mg} / \mathrm{dL}$ in comparison to Group B which was $115.82 \pm 32.46 \mathrm{mg} / \mathrm{dL}$ $(p=0.0002)$. Protective lipid i.e. HDL profile was significantly higher in Group B (controls) in comparison to Group A (cases). Mean HDL cholesterol among Group B was $42.56 \pm$ $6.45 \mathrm{mg} / \mathrm{dL}$, while in Group A (cases) mean HDL was $37.90 \pm 8.16 \mathrm{mg} / \mathrm{dL}(\mathrm{p}=0.0002)$. The lipid profile distribution is detailed in Table 2.

Table 2. Lipid profile distribution among groups $A$ and $B$.

\begin{tabular}{|l|c|c|l|}
\hline \multicolumn{1}{|c|}{ Lipid profile $(\mathbf{m g} / \mathbf{d L})$} & $\begin{array}{c}\text { Group A } \\
\mathbf{( n = 1 1 0 )}\end{array}$ & $\begin{array}{c}\text { Group B } \\
\mathbf{( n = 5 0 )}\end{array}$ & \multicolumn{1}{c|}{ p value } \\
\hline Total cholesterol & $210.09 \pm 0.09$ & $186.62 \pm 31.67$ & 0.0001 \\
\hline Triglyceride & $162.82 \pm 25.08$ & $145.36 \pm 27.83$ & 0.0002 \\
\hline Low-density lipoprotein & $140.31 \pm 39.27$ & $115.82 \pm 32.46$ & 0.0002 \\
\hline High-density lipoprotein & $37.90 \pm 8.16$ & $42.56 \pm 6.45$ & 0.0002 \\
\hline
\end{tabular}

All data are expressed as mean \pm standard deviation

It was found that total cholesterol was raised in $54 \%$ of cases in comparison to $36 \%$ of controls, triglycerides was raised in $64 \%$ of cases in comparison to $32 \%$ of controls, LDL was on the high side in $54 \%$ of cases in comparison to $36 \%$ of controls and HDL values were lower than normal in $73 \%$ of cases in comparison to $44 \%$ of controls.

\section{Apolipoproteins}

Mean ApoB100 and Apo A-1 were significantly different among Group A and Group B. The ApoB100: Apo A-1 ratio was statistically higher in cases of Group $A$ in comparison to healthy controls of Group B ( $\mathrm{p}=0.004)$ as shown in Figure 1.
Apo B100/Apo A1 in groups A \& B

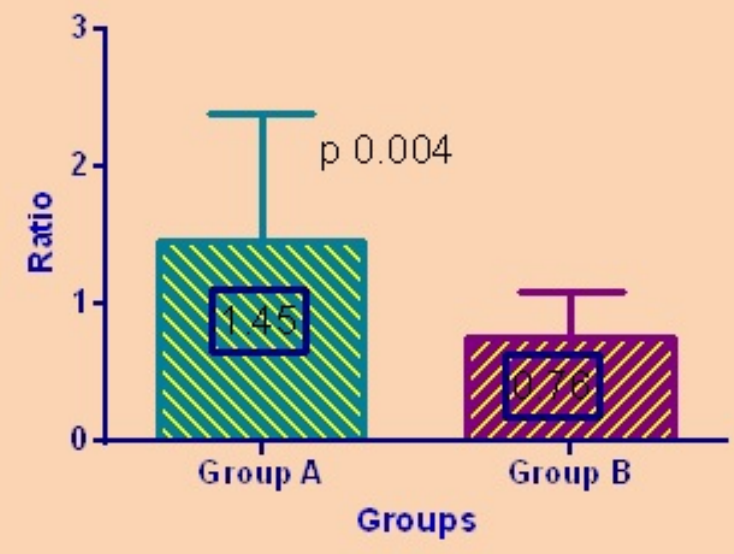

Figure 1. Mean values of ApoB100: Apo A-1 ratio.

ApoB100 and increased ApoB100: Apo A-1 ratio were associated with an increased risk of future cardiovascular events and were raised in $54 \%$ and $57 \%$ of the siblings in comparison to $32 \%$ and $39 \%$ in controls, respectively. Apo A-1 which was found to be protective for cardiovascular events was lower than normal in $52 \%$ of siblings in comparison to $32 \%$ in controls. $\mathrm{Lp}$ (a) was found to be significantly increased in Group A as compared to Group B ( $\mathrm{p}=0.01)$. $\mathrm{Lp}(\mathrm{a})$ was found to above normal range in $60 \%$ of cases in comparison to $38 \%$ in controls. The apolipoprotein profile distribution among the two groups is given in Table 3 .

Table 3. Apolipoprotein profile distribution among groups $A$ and $B$.

\begin{tabular}{|l|l|l|l|}
\hline \multicolumn{1}{|c|}{ Lipid profile $(\mathbf{m g} / \mathbf{d L})$} & Group A $(\mathbf{n = 1 1 0 )}$ & Group B (n=50) & \multicolumn{1}{|c|}{ p value } \\
\hline ApoB100 & $152.17 \pm 51.09$ & $122.32 \pm 18.79$ & 0.003 \\
\hline Apo A-1 & $134.92 \pm 55.61$ & $172.42 \pm 49.84$ & 0.0001 \\
\hline ApoB100: ApoA-1 & $1.45 \pm 0.93$ & $0.76 \pm 0.32$ & 0.004 \\
\hline Lipoprotein (a) & $27.36 \pm 16.24$ & $16.70 \pm 5.93$ & $<0.0001$ \\
\hline
\end{tabular}

All data are expressed as mean \pm standard deviation

\section{Discussion}

In the present study, index patients up to 45 years of age were selected, according to criteria in literature, which considers a patient with acute MI young if he or she is less than 45 years old. To determine the actual prevalence of conventional risk factors, including lipid and lipoprotein abnormalities, 110 coronary disease-free siblings of 100 Indian patients who had documented acute MI before 45 years of age were studied. To compare the results with the general population, 50 healthy controls were selected. In this study, to minimize bias, both age and sex distribution was kept equal without statistically significant differences among cases and controls. Number of male patients were more in comparison to female patients in both the groups, approximately in 2:1 fashion. 
Among risk factors, smoking continues to be a predominant risk factor among siblings in comparison to healthy controls. Majority of male siblings (brothers) were smokers in comparison to female siblings (sisters). Among other risk factors hypertension, diabetes mellitus and increased waist circumference were also significantly more prevalent in siblings than controls. Of the study population, smokers comprised $72 \%$ siblings, hypertensives comprised $42 \%$ siblings, diabetics comprised $30 \%$ siblings and $41 \%$ siblings had an increased waist circumference. These findings are consistent with the results of a study done by Becker et al. [23] to determine the actual prevalence of hyperlipidemia, hypertension and diabetes, and the awareness of these coronary risk factors in unaffected family members. 150 coronary disease-free siblings of 86 people who had documented coronary disease before 60 years of age were studied. With the use of nationally established recommendations for blood pressure and lipids, which are based on coronary disease risk curves, screening revealed that $48 \%$ of brothers and $41 \%$ of the sisters were hypertensive, $45 \%$ of brothers and $22 \%$ of the sisters had a lipid abnormality, $38 \%$ of siblings were current cigarette smokers and $4.7 \%$ were diabetic.

On analysis of lipid profile among siblings and controls it was found that dyslipidemia was significantly more common in siblings as compared to healthy controls. It was found that total cholesterol were raised in $54 \%$ of siblings in comparison to $36 \%$ in controls, triglycerides were raised in $64 \%$ of siblings in comparison to $32 \%$ in controls, LDL was on higher side in $54 \%$ of siblings in comparison to $36 \%$ in controls and HDL was lower than normal in $73 \%$ of siblings in comparison to $44 \%$ in controls. Triglyceride and HDL abnormalities were markedly different in siblings and controls. Mean total cholesterol, triglyceride, LDL and HDL were also significantly different between the siblings and controls. These findings were well matched with other studies which stated that family history of premature CAD in family and familial dyslipidemia are independent future predictors of cardiovascular disease. Surprisingly, the awareness of elevated lipid levels was low in siblings with $73 \%$ unawareness which emphasizes the importance of familial screening of cardiovascular disease (CVD) risk factors [24].

On further analysis of apolipoprotein and $\mathrm{Lp}(\mathrm{a})$ in siblings and controls it was found that values of ApoB100, APO-A1, ApoB100: Apo-A1 ratio and $\mathrm{Lp}$ (a) were significantly different in siblings in comparison to healthy controls. ApoB100 and increased ApoB100: Apo-A1 ratio which were associated with an increased risk of future cardiovascular events were raised in $54 \%$ and $57 \%$ of the patients in comparison to $32 \%$ and $39 \%$ in controls, respectively. Apo-A1 which was found protective for cardiovascular events was lower than normal in $52 \%$ of siblings in comparison to $32 \%$ in controls. These findings are consistent with AMORIS study [25] which stated that increased ratio of the ApoB100: Apo-A1 which were associated with an increased risk of future cardiovascular and cerebrovascular events.

Similarly, $\mathrm{Lp}(\mathrm{a})$ values were also significantly raised in siblings in comparison to controls. $\mathrm{Lp}$ (a) was found to be above normal range in $60 \%$ of siblings in comparison to $38 \%$ in controls. Mean values of these parameters were significantly different in siblings than controls. Surprisingly, there was no significant difference among male and female siblings in relation to these parameters signifying that both sexes are equally at risk for future cardiovascular events in case of history of acute MI in their siblings at a young age and both brothers and sisters need equal attention in relation to risk factor modification and change in lifestyle. These findings are in accordance with the study done by Aila et al [26]. in who concluded that familial hyperlipidemia was twice and familial hypertension three times as common in case as in reference families; other risk factors were equally common in both. It is concluded that most of the familial aggregation of coronary heart disease is mediated by familial aggregations of hyperlipidemia and hypertension.

\section{Study limitations}

This study has a few limitations. The important ones which deserve mention are outlined. First is the study design. This was a case-control non-randomized study. Second is that incidence of risk factors for atherosclerosis were higher in siblings as compared to controls, which may adversely affect the lipid and lipoprotein abnormalities. So, a randomized study with matching atherosclerotic risk factor profile in siblings and control is needed to draw a definite conclusion. Last is the sample size. A larger number of cases is required to draw a definite conclusion on this point.

\section{Conclusion}

Lipid and lipoprotein abnormalities in the form of raising levels of total cholesterol, LDL cholesterol, triglyceridse, decreased levels of HDL cholesterol, increased Apo B: ApoA1 ratio and $L p(a)$ were significantly prevalent in siblings of young acute MI in comparison to the general population. Similarly conventional atherosclerotic risk factors, smoking, hypertension, diabetes mellitus, and increased abdominal obesity were also found to be significantly more prevalent in siblings of young acute MI than the general population. These findings emphasized the importance of familial screening of $\mathrm{CAD}$ risk factors and may be an answer to the possible cause of familial clustering in young MI and an intensive efforts should be made to identify and alter modifiable risk factors in these cases.

\section{References}

1. Lloyd-Mostyn R. National Service Framework for Coronary Heart Disease. BMJ.2000; 321(7261): 634.

2. Office of National Statistics. Indicators of the nation's health-ischemic heart disease: male and female death rates by special causes. 2002 .

3. Office of National Statistics. Weekly incidence of heart attacks.

4. Sinha R. Fisch G, Teague B, et al. Prevalence of impaired glucose tolerance among children and adolescents with marked obesity. N Engl J Med.2002;346(11):802-10. 
5. Conti RAS, Solimene MC, Lemos da Luz P, et al. Comparison Between Young Males and Females with Acute Myocardial Infarction. Arq Bras Cardiol. 2002 ; 79(5):510-25.

6. Friedlander Y, Siscovick DS, Weinmann S, et al. Family history as a risk factor for primary cardiac arrest. Circulation. 1998;97(2):155-60.

7. Leander K, Hallqvist J, Reuterwall C, et al. Family history of coronary heart disease, a strong risk factor for myocardial infarction interacting with other cardiovascular risk factors: Results from the Stockholm Heart Epidemiology Program (SHEEP). Epidemiology. 2001;12(2):215-21.

8. Barrett-Connor E, Khaw K. Family history of heart attack as an independent predictor of death due to cardiovascular disease. Circulation. 1984; 69(6):1065-69.

9. Shea S, Ottman R, Gabrieli C, et al. Family history as an independent risk factor for coronary artery disease. J Am CollCardiol. 1984;4(4): 793-801.

10. Conroy RM, Mulcahy R, Hickey N, et al. Is a family history of coronary heart disease an independent coronary risk factor? Br Heart J. 1985; 53(4):378-81.

11. Slack J. Risks of ischemic heart-disease in familial hyperlipoproteinaemic states. Lancet 1969;2(7635): 1380-82.

12. Phillips RL, Lilienfeld AM, Diamond EL, et al. Frequency of coronary heart disease and cerebrovascular accidents in parents and sons of coronary heart disease index cases and controls. Am J Epidemiol. 1974; 100(2):87-100.

13. Rissanen AM. Familial aggregation of coronary heart disease in a high incidence area (North Karelia, Finland). Br Heart J. 1979; 42(3):294-303.

14. Slack J, Evans KA. The Increased Risk of Death from Ischaemic Heart Disease in First Degree Relatives of 121 Men and 96 Women with Ischaemic Heart Disease J MED Genet. (1966);3(4):239-57.

15. Allen JK, Young DR, Blumenthal RS. et al. Prevalence of hypercholesterolemia among siblings of persons with premature coronary heart disease: application of the Second Adult Treatment Panel guidelines. Arch Intern Med. 1996;156(15):1654-60.

16. Rosseneu M, Fruchart JC, Bard JM, et al. Plasma apolipoprotein concentrations in young adults with a parental history of premature coronary heart disease and in control subjects. The EARS study. European atherosclerosis research study. Circulation.1994; 89(5): 1967-73.

17. Dobson AJ, Alexander HM, et al. How soon after quitting smoking does risk of heart attack decline? J Clin Epidemiol.1991;44(11):1247-53.
18. Chobanian AV, Bakris GL, Black HR, et al. Seventh Report of the Joint National Committee on Prevention, Detection, Evaluation, and Treatment of High Blood Pressure. Hypertension.2003;42:(6)1206-1252.

19. American Diabetes Association. Standards of medical care in diabetes - 2013. Diabetes Care. 2013;36 (Suppl 1): 11-66.

20. National Institutes of Health, National Heart, Lung, and Blood Institute. Clinical guidelines on the identification, evaluation, and treatment of overweight and obesity in adults: the evidence report. Obes Res. 1998;6:51-210.

21. National Cholesterol Education Program (NCEP) Expert Panel on Detection, Evaluation, and Treatment of High Blood Cholesterol in Adults (Adult Treatment Panel III). Third Report of the National Cholesterol Education Program (NCEP) Expert Panel on Detection, Evaluation, and Treatment of High Blood Cholesterol in Adults (Adult Treatment Panel III) final report. Circulation. 2002;106(25):3143-421.

22. Thygesen K, Alpert JS, Jaffe AS, et al. Third Universal Definition of Myocardial Infarction. Circulation. 2012;12(6):2020-2035.

23. Becker DM, Becker LC, Pearson TA, et al. Risk Factors in Siblings of People With premature Coronary Heart Disease. J AmColl Cardiol.1988;12(5):1273-80.

24. McPherson R, Frohlich J, Fodor G, et al. Canadian Cardiovascular Society. Canadian Cardiovascular Society position statement: recommendations for the diagnosis and treatment of dyslipidemia and prevention of cardiovascular disease. Can J Cardiol. 2006; 22:(11)913-27.

25. Walldius G, Jungner I, Kolar W, et al. High cholesterol and triglyceride values in Swedish males and females: increased risk of fatal myocardial infarction: First report from the AMORIS (Apolipoprotein related MOrtalityRISk) Study. Blood Press 1992;4: 35-42.

26. AM Rissanen, Nikkila EA. Aggregation of coronary risk factors in families of men with fatal and non-fatal coronary heart disease. Br Heart J. 1979; 42(4):373-80.

\section{*Correspondence to}

Awadhesh Kumar Sharma

Deparment of Cardiology

LPS Institute of Cardiology

Kanpur, UP

India

E-mail: awakush@gmail.com 